\title{
Experimental study on advanced bone regeneration by BMP-7 derived-peptide chitosan nanometer hydroxyapatite collagen biomimetic composite
}

\author{
Bo Feng*1,2, Dongxu Hu${ }^{1}$, Yangde Zhang ${ }^{1}$ \\ ${ }^{1}$ Institute of Biomedical Engineering, Central South University, Changsha, Hunan, China \\ ${ }^{2}$ Department of Orthopedics, The Third Affiliated Hospital of Inner Mongolia Medical University, Baotou, Inner Mongolia, \\ China
}

Received: March 21, 2016

DOI: $10.14725 /$ dcc.v3n2p16
Accepted: May 8, 2016

URL: http://dx.doi.org/10.14725/dcc.v3n2p16

\begin{abstract}
Objective: To investigate the effect of BMP-7 derived-peptide chitosan nanometer hydroxyapatite biomimetic collagen composite on repairing rat critical-sized cranial defects.

Methods: The chitosan nanometer hydroxyapatite collagen composite was prepared and the microcosmic appearance of the composite was observed by scanning electron microscope. The BMP-7 derived-peptide was introduced into the composite by vacuum adsorption. The released peptide content from the scaffold was detected using high performance liquid chromatography at different set times. Critical-sized cranial defects were created on both sides of the parietal bone in 24 adult Sprague-Dawley rats. The BMP-7 derived-peptide chitosan nanometer hydroxyapatite biomimetic collagen composites were implanted on the right side as experimental group and the left side was implanted with chitosan nanometer hydroxyapatite biomimetic collagen composites alone as control group. The rats of both groups were killed in batch respectively after 6 and 12 weeks. Macroscopic observation, three-dimensional reconstruction of computed tomography (CT) and histological observation were performed on these samples.

Results: The results of scanning electron microscope showed that the surface of the scaffold was porous. The releasing character of BMP-7 derived-peptide belonged to slow release. The result of animal experiment showed that the BMP-7 derived-peptide chitosan nanometer hydroxyapatite biomimetic collagen composite could more effectively promote the repair of cranial bone defects comparing with the chitosan nanometer hydroxyapatite biomimetic collagen composite alone. The difference was statistically significant $(p<.05)$.

Conclusions: The BMP-7 derived-peptide chitosan nanometer hydroxyapatite collagen biomimetic composite can effectively promote bone regeneration of bone defects. The composite is a kind of ideal scaffold material for bone tissue engineering.
\end{abstract}

Key Words: BMP-7 derived-peptide, Scaffold material, Cranial defects, Bone tissue engineering

The treatment of bone defects has always been a puzzle for orthopedic surgeons. At present, autologous bone and allogenic bone transplantation are mainly used to treat bone defects. However, these methods are inadequate to meet the need of clinically treating bone defects. Therefore, the development of artificial bone repair materials with good biocompatibility, biodegradability, bone conduction and osteoinduction has been a hot research topic in the field of bone defect treatment. ${ }^{[1-3]}$ In this study, chitosan nanometer hydroxyapatite collagen composite material was prepared according to the bionics principle. Combined with osteoinductive BMP-7 derived-peptide, the BMP-7 derived-

*Correspondence: Bo Feng; E-mail: dcc58@ncspress.com; Address: Department of Orthopedics, The Third Affiliated Hospital of Inner Mongolia Medical University, Baotou, Inner Mongolia, China. 
peptide chitosan nanometer hydroxyapatite biomimetic collagen composite was prepared and implanted into the skull defects in rats to evaluate its ability to promote bone regeneration.

\section{Materials and methods}

\subsection{Materials and reagents}

Twenty-four Sprague-Dawley (SD) rats, two-grade cleanness, male and female, aged 30-35 days (average 32 days), weighing 100-120 g (average $110 \mathrm{~g}$ ), healthy, were kept in a clean environment. BMP-7 derived-peptide (Shanghai Jill Pharmaceutical Co., Ltd.), collagen (Wuhan Asian Pharmaceutical Co., Ltd.), others were all domestic analytical reagents. SENSATION 16 type computerized tomography (SIEMENS, Germany), high-performance liquid chromatography (HPLC) (GIAPO).

\subsection{Methods}

\subsubsection{Preparation of BMP-7 derived-peptide chitosan nanometer hydroxyapatite biomimetic collagen composite}

An amount of $10 \mathrm{~g}$ collagen was taken in the $0.5 \mathrm{M}$ acetic acid solution of $200 \mathrm{~mL}$, and it was stirred evenly to be fully dissolved. In order to make the $\mathrm{pH}$ value of solution 7.4, $0.5 \mathrm{M} \mathrm{H}_{3} \mathrm{PO}_{4}$ solution and $0.5 \mathrm{M} \mathrm{CaCl}_{2}$ solution along with $0.5 \mathrm{M} \mathrm{NaOH}$ solution were slowly added to the above collagen solution. Then, the solution was fully stirred for more than $12 \mathrm{~h}$, and the precipitated products were lapping into powder after freezing. The appropriate amount of chitosan was dissolved in hexafluoroisopropanol organic solvent to prepare a viscous solution with a mass volume ratio of $10 \%$. A certain amount of nanometer hydroxyapatite collagen powder was added and dispersed by ultrasonic wave for $30 \mathrm{~min}$. After stirring well, cooling, forming, and then freeze-dried to remove organic solvents for use. The micromorphology of the surface of the composite was observed by scanning electron microscope.

The nanometer hydroxyapatite collagen composite material was made into a cylinder with a diameter of $5 \mathrm{~mm}$ and a thickness of $2 \mathrm{~mm}$, soaked in $1 \mathrm{~mL}$ of polypeptide solution with a concentration of $1 \mathrm{mg} / \mathrm{mL}$ for $30 \mathrm{~min}$ and vacuum-adsorbed for $30 \mathrm{~min}$ so that the amount of polypeptide loaded on each material was $1 \mathrm{mg}$.

\subsubsection{Release rules of BMP-7 derived-peptide in vitro}

The scaffolds of 5 polypeptides were immersed in $2 \mathrm{~mL}$ PBS and placed in a $37^{\circ} \mathrm{C}$ incubator at different time points to extract the release solution, wherein the content of BMP-7 polypeptide was detected by HPLC and the cumulative release was calculated.

\subsubsection{Animal experiments}

Twenty-four adult SD rats were randomly divided into 2 groups at 2 time points of 6 and 12 weeks, with 12 animals in each group. The rats were intraperitoneally injected with $2 \%$ pentobarbital sodium of $40 \mathrm{mg} / \mathrm{kg}$. The skin, subcutaneous and periosteum of the middle line of the rat skull were cut respectively, with the drill on the left and right parotid diameter of $5 \mathrm{~mm}$ round-shaped skull full-thickness bone defects. The BMP-7 derived-peptide chitosan nanometer hydroxyapatite biomimetic collagen composites were implanted on the right side as experimental group and the left side was implanted with chitosan nanometer hydroxyapatite biomimetic collagen composites alone as control group.

\subsubsection{Materials and testing}

Animals in each group were sacrificed at 6 and 12 weeks after operation, respectively. The specimens were removed for the following tests: (1) General observation of the specimens: The degradation of implanted material and the repair of bone defects were observed visually. (2) CT reconstruction: Three dimensional reconstruction was carried out after CT scanning, and the repair of bone defect was observed. (3) HE staining: After fixed with $4 \%$ paraformaldehyde, the tissues were decalcified with EDTA and paraffinembedded for HE staining. Three slices of each specimen and two visual fields of slice were taken, and the percentage of bone area per field was calculated by Image-Proplus 5 image analysis software. The difference of bone area between the experimental group and the control group was compared.

\subsection{Statistical analysis}

Data were analyzed and processed using SPSS 13.0 statistical software. The data were expressed as mean \pm standard deviation $(\bar{x} \pm \mathrm{s})$, and the independent sample $t$-test was used to compare the two groups.

\section{Results}

\subsection{Microstructure of chitosan nanometer hydrox- yapatite biomimetic collagen composite}

The results of electron microscopy showed that the composite was a porous structure with an aperture of about 10-100 $\mu \mathrm{m}$ (see Figure 1). 


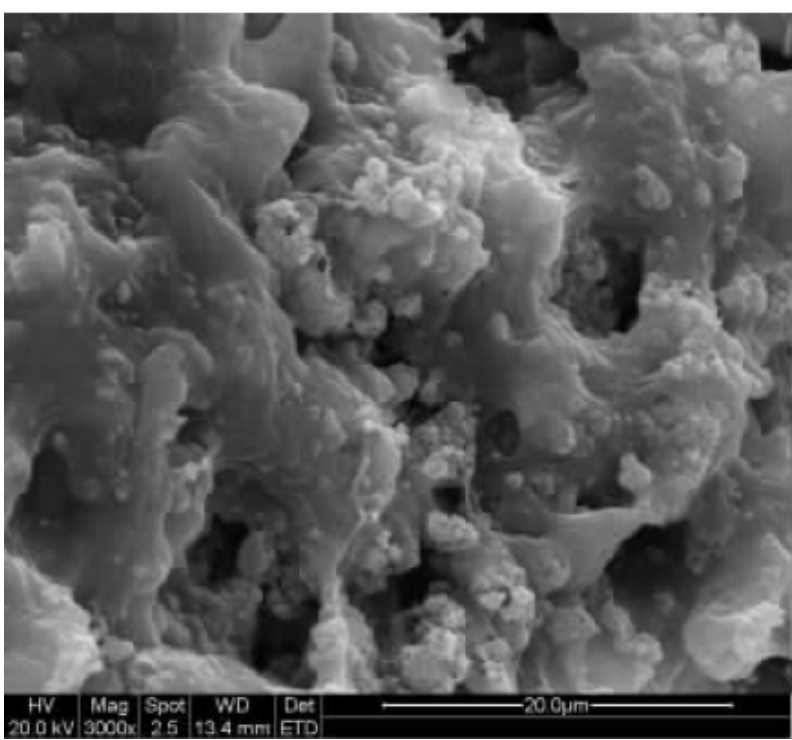

Figure 1: Micro morphology of chitosan nanometer hydroxyapatite biomimetic collagen composite

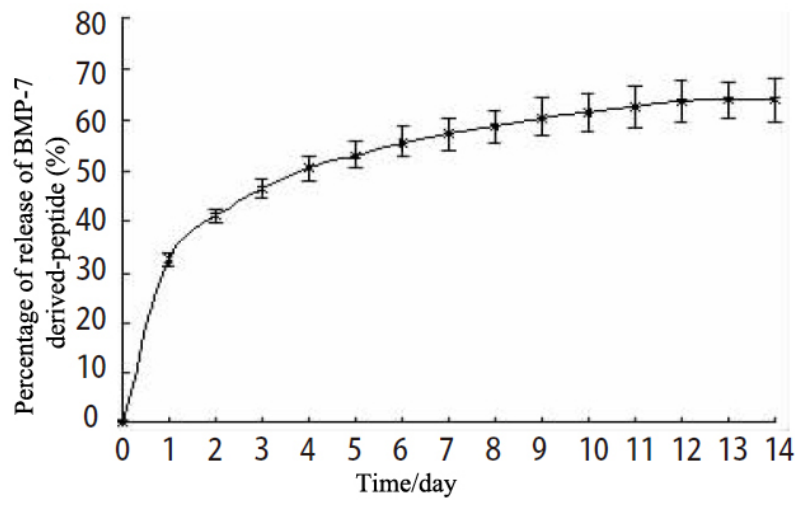

Figure 2: Release of BMP-7 derived-peptide at different time points

\subsection{Release rules of BMP-7 derived-peptide in vitro}

The results of in vitro release of BMP-7 derived-peptide were shown in Figure 2. The released amount of BMP-7 derived-peptide on day 1 was about $32.5 \% \pm 1.22 \%$ of the total amount, which was an explosive release, the cumulative amount of 14 days accounted for $(64.02 \pm 4.21) \%$ of the total.

\subsection{Animal experiment results}

After 6 weeks, the implanted material was closely connected with the edge of the bone defect, and the boundary was blurred, and the texture of the implant was slightly hard. The three-dimensional reconstruction of CT showed that there were some flaky high density shadows in the visible defect area, and the density was similar to that of normal bone.
However, the boundary between the implant material and the bone defect in the control group was observed to be obvious. In three-dimensional reconstruction of $\mathrm{CT}$, there was only a slight flake dense shadow in the visible defect area, which was lower than the normal bone density (see Figure 3).

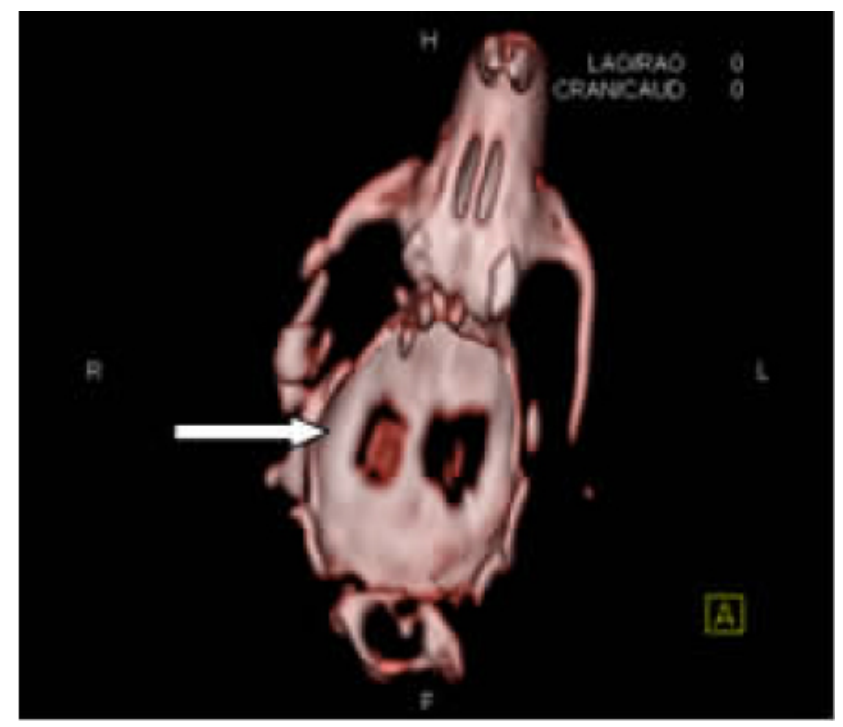

Figure 3: Three-dimensional reconstruction of CT results of specimens in the experimental group (white arrow) and the control group after 6 weeks of operation

Histological observation showed that a large number of osteoblasts grew into the materials, some have begun to degrade. In the control group, only a small amount of osteoblasts were seen inside the materials (see Figure 4).

12 weeks after operation, the experimental group showed that the implant materials were basically replaced by regenerated bone tissue, and the texture was hard. The threedimensional reconstruction of CT showed that the defect was completely healed, and the marginal boundary between the implant material and the bone defect was obvious in the control group. Three-dimensional reconstruction of CT showed enhanced high density, and the area close to the defect was $1 / 2$, failed to completely repair the bone defect (see Figure 5).

Histological observation of the experimental group showed that most of the implanted materials were degraded and replaced by mature lamellar bone, leaving a small amount of braided bone. In the control group, only a few osteoblasts were seen inside the materials during the histological observation (see Figure 6).

The percentages of new bone formation in the experimental group and the control group at 6 weeks and 12 weeks after surgery were shown in Figure 7 . The percentage of new bone formation area at each time point in the experimental group was significantly higher than that in the control group $(p<.05)$. 

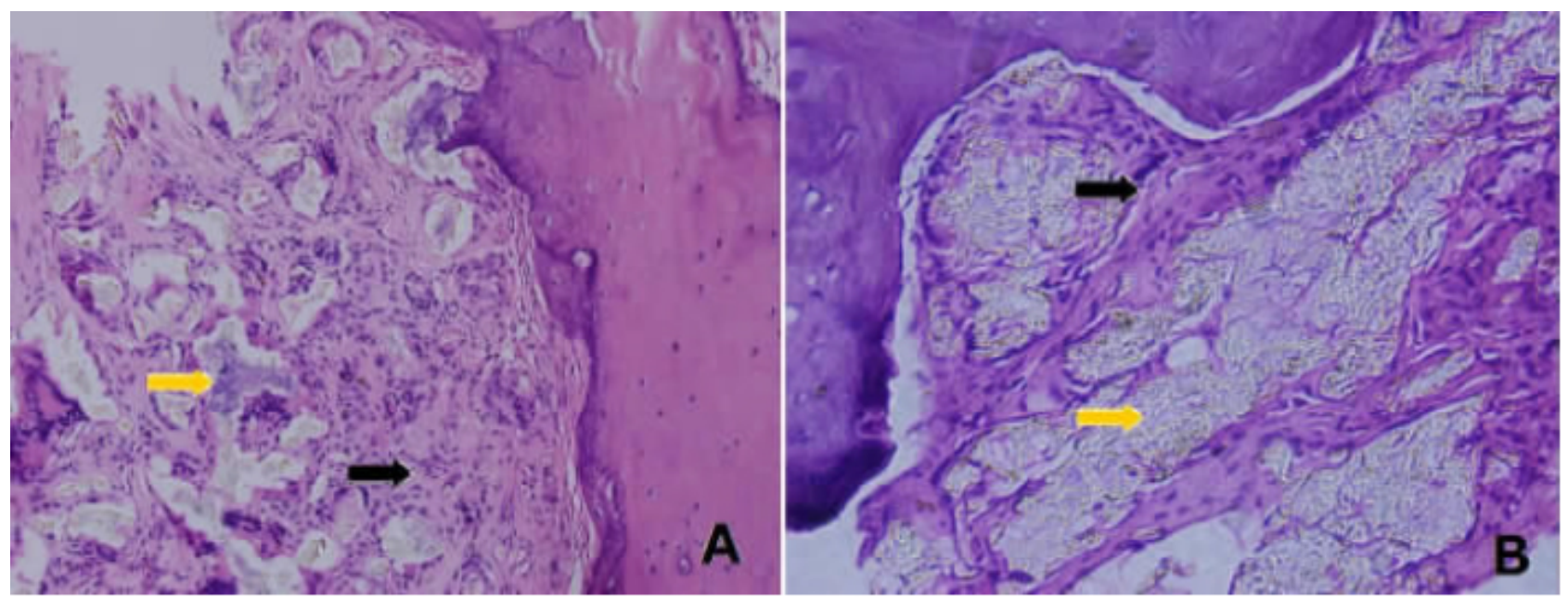

Figure 4: HE staining after 6 weeks of operation

A: The materials had started to degrade in the experimental group after 6 weeks of operation, and a large number of osteoblasts grew inside the materials. The yellow arrow referred to the material, and the black arrow referred to the new bone (200 times magnified under the light microscope). B: HE staining of the control group after 6 weeks of operation. It could be seen that a small amount of osteoblasts grew into the interior of the material, and the yellow arrowhead was shown as the materials, and the black arrowhead was shown as a new bone tissue (200 times magnified under the light microscope)

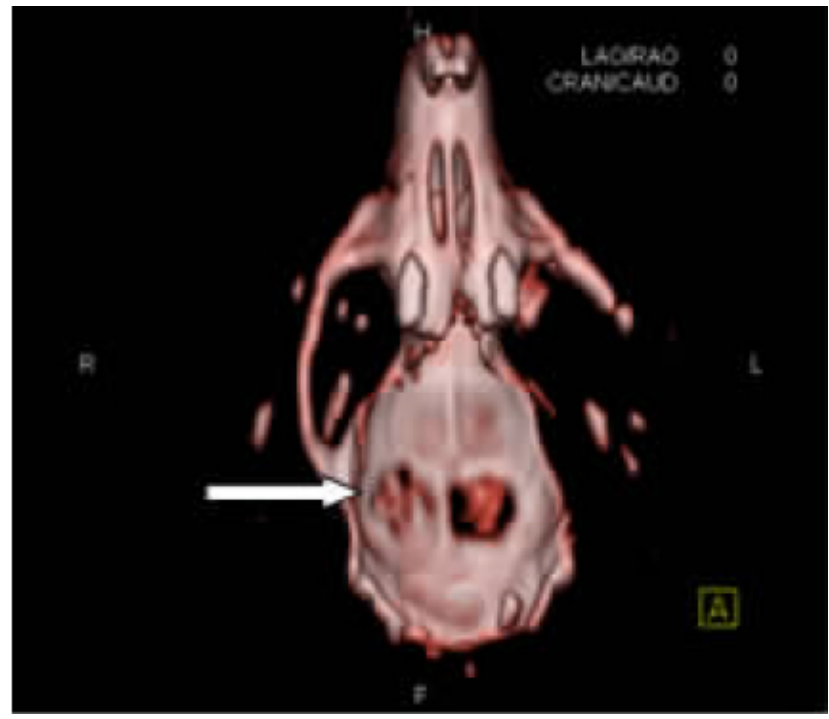

Figure 5: CT reconstruction results of experimental group (indicated by white arrow) and control group at 12 weeks after operation

\section{Discussion}

Ideal bone repair materials should meet the following requirements: (1) three-dimensional porous structure; (2) good biocompatibility; (3) good biodegradability, material degradation rate and new bone regeneration speed matching; (4) good plasticity and mechanical strength; (5) good osteoPublished by New Century Science Press conductivity; (6) good osteoinduction; (7) material handling of various properties. For example, the change of the material degradation rate, mechanical strength, porosity and pore size could be made according to clinical needs. ${ }^{[4-7]}$ To date, no artificial bone repair material has been recognized by the scientific community. However, biomimetic-based composite bone repair materials have gained wide attention due to their good biocompatibility, degradability and osteoconductivity. ${ }^{[8,9]}$ The chitosan nanometer hydroxyapatite collagen biomimetic composite which was prepared in our study is a kind of these materials. Scanning electron microscopy results showed that the composite was a loose porous structure similar to the porous structure of natural bone tissue. In order to improve the osteoinductivity of this composite material, we designed and synthesized a novel BMP-7 peptide with 13 amino acids, which was combined with chitosan nanometer hydroxyapatite collagen materials composite, in order to build a new type of bionic material BMP-7 derivedpeptide chitosan nanometer hydroxyapatite collagen. BMP7 derived-peptide is a novel active molecule with similar biological activity to BMP-7. At the same time, its spatial structure is simple and spiral, and the active site is easy to expose. We found it easy to mix with the scaffold material. It is generally believed that only after bioactive factors are released from scaffold materials, biological active factors, then, can be released slowly from materials to maximize their biological functions. ${ }^{[10]}$ In this study, the release rules of BMP-7 derived-peptide were detected in the materials. The results showed that the BMP-7 derived-peptide was actively released on the first day and then released slowly, which basically met the requirement of sustained release. 

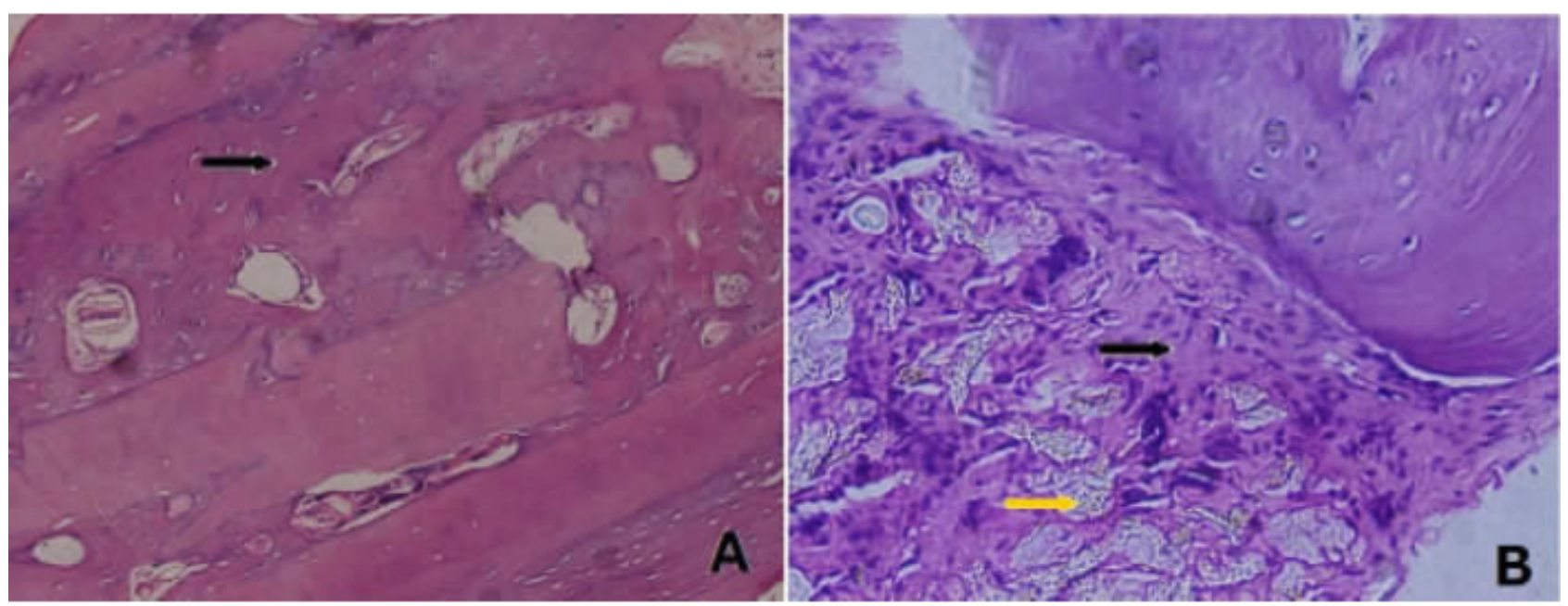

Figure 6: HE staining after 12 weeks of operation

A: After 12 weeks of operation, HE staining showed that most of the implanted materials were degraded and replaced by mature lamellar bone, leaving a small amount of braided bone (200 times magnified under the light microscope). B: After 12 weeks of operation, the control group was stained with HE, which showed that the materials were partially degraded and scattered in the immature braided bone (200 times magnified under the light microscope)

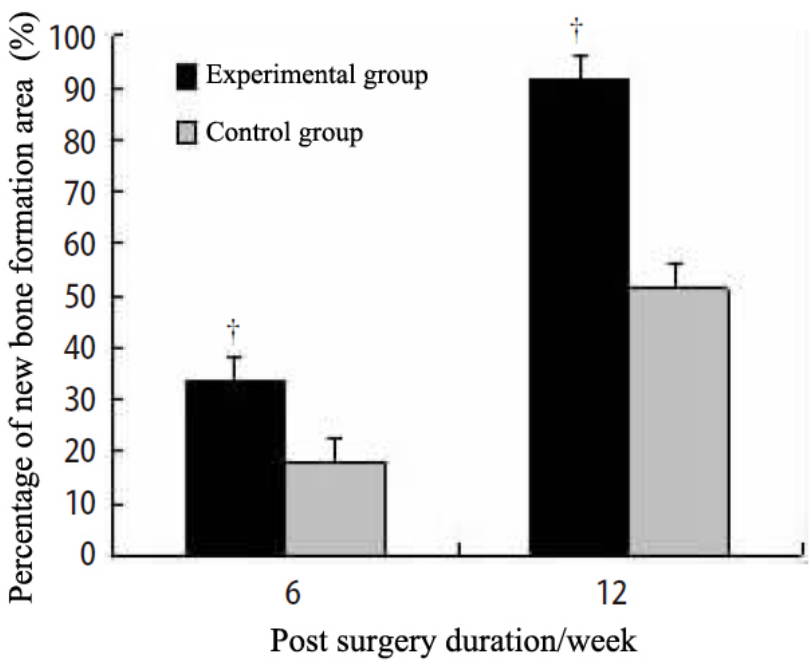

$\doteqdot P<0.05$

Figure 7: The area percentage of new bone formation in experimental group and control group at each time point

In order to evaluate the ability of BMP-7 derived-peptide chitosan nanometer hydroxyapatite collagen composite to promote bone regeneration, we select the rat skull defect as experimental animal model, which is characterized by the advantages of simple, standard and reproducible. ${ }^{[11]}$ Based on the results of the preliminary experiment, the researchers selected $1 \mathrm{mg}$ as the amount of the composite polypeptide, and polypeptide chitosan nanometer hydroxyapatite biomimetic collagen composites and pure chitosan nanometer hydroxyapatite biomimetic collagen composites alone were respectively implanted into the rat skull defects. General observation, three dimensional reconstruction of CT and histological observation were performed at 6 and 12 weeks after the operation. The results showed that the osteogenic capacity of BMP-7 derived-peptide chitosan nanometer hydroxyapatite biomimetic collagen composite was significantly stronger than the chitosan nanometer hydroxyapatite biomimetic collagen composite alone, and the difference was significant. It indicated that BMP-7 derived-peptide significantly improved the bone inducibility of chitosan nanometer hydroxyapatite biomimetic collagen composites. The new biomimetic scaffold material BMP-7 derived-peptide chitosan nanometer hydroxyapatite biomimetic collagen is a promising biomimetic bone repair material.

\section{Conflicts of Interest Disclosure}

The authors have no conflicts of interest related to this article.

\section{References}

[1] Carson JS, Bostrom MP. Synthetic bone scaffolds and fracture repair. Injury. 2007; 38(Suppl 1): S33-7. PMid: 17383484. https: //doi.org/10.1016/j.injury.2007.02.008

[2] Hench LL, Polak JM. Third-generation biomedical materials. Sci- ence. 2002; 295: 1014-1017. PMid: 11834817. https://doi. org/10.1126/science.1067404

[3] Wlodarski KH, Wlodarski PK, Galus R. Bioactive composites for bone regeneration. Review. Ortop Traumatol Rehabil. 2008; 10(3): 201-210. PMid: 18552757.

[4] Rezwan K, Chen QZ, Blaker JJ, et al. Biodegradable and bioactive 
porous polymer/inorganic composite scaffolds for bone tissue engineering. Biomaterials. 2006; 27(18): 3413-3431. PMid: 16504284 https://doi.org/10.1016/j.biomaterials.2006.01.039

[5] Ge Z, Jin Z, Cao T. Manufacture of degradable polymeric scaffolds for bone regeneration. Biomed Mater. 2008; 3(2): 022001. PMid: 18523339. https://doi.org/10.1088/1748-6041/3/ 2/022001

[6] Karageorgiou V, Kaplan D. Porosity of 3D biomaterial scaffolds and osteogenesis. Biomaterials. 2005; 26(27): 5474-5491. PMid: 15860204. https://doi.org/10.1016/j.biomaterials. 2005.02 .002

[7] Scott TG, Blackburn G, Ashley M, et al. Advances in bionanomaterials for bone tissue engineering. J Nanosci Nanotechnol. 2013; 13(1): 1-22. PMid: 23646693. https://doi.org/10.1166/ jnn.2013.6733

[8] Swetha M, Sahithi K, Moorthi A, et al. Biocompositescontaining natural polymers and hydroxyapatite for bone tissueengineering. In- ternational Journal of Biological Macromolecules. 2010; 47(1): 14. PMid: 20361991. https://doi.org/10.1016/j.ijbiomac. 2010.03 .015

[9] Kim SS, Ahn KM, Park MS, et al. A poly(lactide-co-glycolide)/hydroxyapatite composite scaffold with enhanced osteoconductivity. J Biomed Mater Res A. 2007; 80(1): 206-215. PMid: 17072849. https://doi.org/10.1002/jbm.a.30836

[10] White LJ, Kirby GT, Cox HC, et al. Accelerating protein release from microparticles for regenerative medicine applications. Mater Sci Eng C Mater Biol. 2013; 33(5): 2578-2583. PMid: 23623071. https://doi.org/10.1016/j.msec.2013.02.020

[11] Develioglu H, Unver Saraydin S, Kartal U. The bone-healing effect of a xenograft in a rat calvarial defect model. Dent Mater J. 2009; 28(4): 396-400. PMid: 19721275. https://doi.org/10.4012/ $\mathrm{dmj} \cdot 28.396$ 